\title{
Dificuldades para atividades básicas e instrumentais de vida diária, referidas por usuários de um Centro de Saúde Escola do Município de São Paulo
}

\section{Dfficultieswith basicand instu mental ativities of dily living reported by users of a Sthod Health CenterinSz̃oPaloCarty}

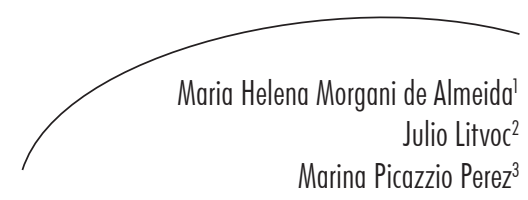

\section{Resumo}

Idosos que atendem a determinados critérios demográficos, sociais e econômicos estão especialmente vulneráveis a adoecimento, incapacidades e morte. Desenvolveu-se estudo com o objetivo de identificar idosos vulneráveis segundo critérios determinados e comparar idosos vulneráveis e não-vulneráveis em relação à necessidade de ajuda para atividades. O estudo, de corte transversal, compreendeu coleta de dados com 190 idosos usuários de um Centro de Saúde Escola, em seus domicílios, entre 2006 e 2008, por meio de aplicação do Instrumento para Classificação de Idosos quanto à Capacidade para o Autocuidado. Cento e quarenta e cinco idosos (80\%) estão submetidos a pelo menos um critério de risco; $99(52,1 \%)$ referem uma ou mais dificuldades para atividades básicas, sendo que 29 (29,3\%) requerem ajuda; 92 (48,4\%) idosos mencionam dificuldades para atividades instrumentais e destes, $67(72,8 \%)$ requerem ajuda. Somente $16(8,4 \%)$ adotam práticas de autocuidado para minimizar suas dificuldades e 38 (20\%) as adotam para compensar parte delas. O grupo de idosos que atende pelo menos a um critério de risco apresenta proporção maior de indivíduos que requer ajuda para atividades básicas e instrumentais de vida diária. Outras análises possibilitarão verificar a influência de cada critério sobre a funcionalidade. A realização de pesquisa sobre práticas de autocuidado com um maior número de indivíduos possibilitará comparação entre grupos que empregam ou não essas práticas e critérios de risco. Apesar das limitações do estudo, ele suscita planejamento de ações aos idosos com dificuldades e que requeiram ou não ajuda para suas atividades.

Faculdade de Medicina, Departamento de Fonoaudiologia, Fisioterapia e Terapia Ocupacional. Universidade de São Paulo. São Paulo, SP, Brasil.

2 Faculdade de Medicina, Departamento de Medicina Preventiva. Universidade de São Paulo. São Paulo, SP, Brasil.

3 Faculdade de Medicina, Departamento de Fonoaudiologia, Fisioterapia e Terapia Ocupacional. Universidade de São Paulo. São Paulo, SP, Brasil.

E-mail:hmorgani@usp.br

Palavras-chave: Idoso. Promoção da Saúde. Autocuidado. Apoio Social. Atividades Cotidianas. 


\section{Abstract}

Elderly who match certain demographic, social and economic criteria are especially vulnerable to illness, disability and death. The study was developed in order to both identify vulnerable elderly according to specific criteria and compare vulnerable and non-vulnerable elderly with their need for assistance with activities. This crosssectional study had its data collected in the household of 190 elderly users of a School Health Center. It took place between 2006 and 2008, through the application of the CICAc Instrument: classification of the aged concerning self-care ability. One hundred and forty-five elderly (80\%) were under at least one risk criterion; 99 (52.1\%) reported one difficulty or more in basic activities, and 29 (29.3\%) of them require assistance; 92 $(48.4 \%)$ elderly mention difficulties in instrumental activities and $67(72.8 \%)$ require assistance. Only $16(8.4 \%)$ adopt self-care practices to minimize their difficulties and $38(20 \%)$ adopt them to compensate just part of their difficulties. The elderly group that is under at least one risk criterion has a higher proportion of individuals who require assistance with basic and instrumental activities of daily living. Further analysis may verify the influence of each criterion on the functionality. Carrying out research on self-care practices with a larger number of individuals will allow a comparison between groups that make or do not make use of these practices and match risk criteria. Despite the limitations of the study, it gives rise to the planning of interventions for the elderly that have difficulties and need assistance with their activities.
Key words: Aged. Health Promotion. Self Care. Social Support. Activities of Daily Living.

\section{INTRODUÇÃO}

O envelhecimento aumenta a susceptibilidade para enfermidades crônicas e incapacidades. De acordo com a Organização Mundial da Saúde (OMS), ${ }^{1}$ estão especialmente vulneráveis ao adoecimento, incapacidades e morte, aqueles que atendem a determinados critérios demográficos, sociais e econômicos: idosos com 80 anos e mais, mulheres idosas, sobretudo solteiras e viúvas, os que moram sozinhos, os socialmente isolados, os sem filhos e aqueles que contam com recursos econômicos muito escassos.

A maior expectativa de vida das mulheres em relação aos homens e o fato de se manterem viúvas em maior proporção as tornam mais vulneráveis a situações como doenças crônicas e incapacidades, pobreza e isolamento social. ${ }^{2}$ Os que sofrem violência física e emocional e aqueles com piores níveis educacionais também se mostram vulneráveis à deterioração da funcionalidade. ${ }^{2}$

Ter limitações graves ou incapacidades e compor casal de idosos no qual um deles é incapacitado ou muito doente, por sua vez, pode determinar ou agravar doenças e associar-se à mortalidade. ${ }^{1}$
A presença de incapacidade tem sido adotada como um importante critério de fragilidade em idosos. A Política Nacional de Saúde da Pessoa Idosa $^{3}$ considera idoso frágil ou em situação de fragilidade aquele que apresenta condições causadoras de incapacidade funcional - como acidente vascular encefálico, síndromes demenciais e outras doenças neurodegenerativas, etilismo, neoplasia terminal, amputações de membros - ou já se encontre com pelo menos uma incapacidade funcional para atividades básicas de vida diária, ou seja, para aquelas relacionadas ao autocuidado físico. Outros critérios estabelecidos pela mesma política para definir condição de fragilidade são: viver situação de violência doméstica; viver em instituição de longa permanência para idosos; encontrar-se acamado e estar hospitalizado recentemente por qualquer razão. Segundo o critério etário, é frágil o idoso com 75 anos ou mais. ${ }^{3}$

O envelhecimento, especialmente em condições de maior fragilidade, gera crescente demanda por serviços médico-sociais, políticas e programas de promoção da saúde e de prevenção de enfermidades e incapacidades. ${ }^{4}$ 
Reconhecendo as necessidades de prevenção e tratamento de condições de fragilidade, a Política Nacional de Saúde do Idoso ${ }^{5}$ tem como propósitos: promoção do envelhecimento saudável, manutenção e melhoria da capacidade funcional, prevenção de doenças, recuperação da saúde e reabilitação, de modo a garantir aos idosos permanência no meio em que vivem, exercendo da forma mais independente possível suas funções na sociedade.

Do ponto de vista da saúde pública, a capacidade funcional, designada como capacidade de manter as habilidades físicas e mentais necessárias para vida independente e autônoma, surge como um novo conceito de saúde, mais adequado para instrumentalizar e operacionalizar a atenção à saúde do idoso. Ações preventivas, assistenciais e de reabilitação devem objetivar, sempre que possível, a manutenção, melhoria ou recuperação desta capacidade. Ao focar a capacidade funcional, transcende-se o simples diagnóstico e tratamento de doenças específicas em direção a preservação da independência física e mental do idoso e valorização de sua autonomia e autodeterminação. ${ }^{5}$

A independência, entendida como a habilidade de executar funções relacionadas à vida diária e de viver em comunidade com alguma ou nenhuma ajuda de outros, envolve capacidade funcional e favorece a autonomia. A independência permite que o indivíduo empreenda ações relativas a suas decisões sobre como se deve viver diariamente, de acordo com suas próprias regras e preferências. ${ }^{2}$

Ações voltadas à capacidade funcional incluem controlar fatores de risco para incapacidades. A fim de manter idosos sadios, independentes e com qualidade de vida, fatores de risco comportamentais e ambientais - de doenças crônicas e declínio funcional devem ser mantidos baixos, e os fatores de proteção, elevados. ${ }^{2}$

Dentre os fatores comportamentais favoráveis a saúde e funcionalidade, destacam-se: envolvimento em atividades físicas adequadas, alimentação saudável, abstinência do fumo e do álcool e uso adequado de medicamentos. Fazem parte dos fatores de proteção ambiental relacionados a saúde e capacidade funcional: proximidade de membros da família, serviços e transporte, adaptações em residências e locais públicos que favoreçam interação e previnam quedas e outros acidentes. ${ }^{2}$

O controle desses fatores configura-se como estratégia para promoção da saúde e compõe, com outras ações, práticas de autocuidado. ${ }^{6}$ A habilidade referida para o autocuidado foi preditor de maior funcionalidade e menor mortalidade entre idosos residentes em uma comunidade.? Reconhece-se a importância de adaptações adotadas por idosos e/ou cuidadores que visam a manter ou aumentar a independência funcional nas atividades de vida diária. Estas, em seu conjunto, são conceituadas como práticas de autocuidado e compreendem basicamente mudanças comportamentais, ambientais e uso de dispositivos auxiliares. ${ }^{8,9}$

Mudanças comportamentais referem-se a técnicas adaptadas para a realização de atividades, sua reorganização no cotidiano ou substituição de antigas atividades por novas. Essas mudanças podem incluir evitar uso de escadas e objetos pesados, fracionar compras, equilibrar realização de atividades que impliquem alta demanda física com outras que envolvam menor consumo de energia, ajustar a realização de atividades às capacidades da pessoa ou torná-las mais confortáveis e seguras.

Modificações ambientais também constituem estratégias compensatórias às dificuldades, abarcando reformas e instalações de barras e corrimãos ou medidas simples como retiradas de obstáculos. Para empreender modificações ambientais, deve-se considerar o significado atribuído pelo idoso ao seu ambiente. Este muitas vezes compõe sua autobiografia, conferindo-lhe segurança e apoio especialmente em situações de perda. Assim, ajustes no ambiente podem ser propostos desde que equilibrem necessidades objetivas e subjetivas.

Para capacitar a pessoa ao desempenho da tarefa, pode-se utilizar ainda dispositivo auxiliar ou de tecnologia assistiva. Esse dispositivo é definido como "qualquer item, 
peça de equipamento ou sistema, quer adquirido comercialmente, modificado ou customizado; usado para aumentar, manter ou melhorar a capacidade funcional de indivíduos com incapacidades." ${ }^{10}$ Como estratégia para promover saúde, especialmente de idosos, considera-se que práticas de autocuidado ${ }^{8,9}$ possam voltarse ainda ao exercício de distintas capacidades, prevenção ou combate ao isolamento social. Nessa perspectiva, as práticas incluem incentiválos a se envolver num universo diversificado e significativo de atividades, manter ou resgatar participação social.

Nesse contexto, desenvolveu-se pesquisa com idosos em seus domicílios por meio da qual se objetivou: a) identificar indivíduos, com 60 anos ou mais que atendam a critérios demográficos e socioeconômicos para adoecimento, incapacidades e morte definidos pela OMS, ${ }^{1}$ que refiram dificuldades para atividades básicas e instrumentais de vida diária e níveis de dificuldade referidos para essas atividades, incluindo necessidade de ajuda; e b) comparar idosos que atendam ou não aos critérios demográficos e/ou socioeconômicos ${ }^{1}$ em relação à necessidade de ajuda para atividades. Propõese apresentar os principais resultados da referida pesquisa.

\section{PROCEDIMENTOS METODOLÓGICOS}

A pesquisa teve apoio da Fapesp e realizada segundo os padrões exigidos pela Declaração de Helsinque, aprovada pelo Comitê de Análise de Projetos de Pesquisa da FMUSP, número
721/05. Desenvolveu-se entre 2006 e 2008 e compreendeu duas visitas a cada idoso em seu domicílio. Nas visitas iniciais, procedeu-se a coleta das informações. Num prazo de uma a duas semanas, após análise das informações, os idosos foram revisitados e foram prestadas orientações quanto ao autocuidado.

Realizou-se estudo, de corte transversal, no qual foram utilizados dados coletados nas visitas iniciais. As visitas aos domicílios foram feitas a 190 indivíduos com 60 anos e mais, residentes em subárea de abrangência do Centro de Saúde Escola Geraldo de Paula Souza da FSP/ USP, usuários do serviço no período de janeiro de 2004 a abril de 2005.

Para a caracterização dos idosos quanto aos critérios demográficos e socioeconômicos, definidos pela OMS para enfermidades, incapacidades e morte $^{1}$ e dificuldades para atividades cotidianas aplicou-se, através de entrevistas individuais, o Instrumento para Classificação de Idosos quanto à Capacidade para o Autocuidado (CICAc). Esse instrumento foi validado em seu conteúdo ${ }^{11}$ e avaliado em sua confiabilidade ${ }^{12}$ para utilização por terapeutas ocupacionais com idosos residentes na comunidade e usuários da atenção primária à saúde, compreendendo quatro dimensões: arranjo doméstico e rede de suporte, breve perfil social e econômico, universo ocupacional e capacidade funcional, com ênfase em práticas de autocuidado ${ }^{8,9}$ para manter ou melhorar essa capacidade. O quadro 1 explicita dimensões, questões e quadros para registros de respostas que compõem o instrumento CICAc. ${ }^{11}$ 
Quadro 1 - Componentes do instrumento CICAc ${ }^{11}$. São Paulo, SP, 2008.

\begin{tabular}{|c|c|}
\hline DIMENSÕES E QUESTÕES & $\begin{array}{l}\text { ACOMPANHA QUADRO PARA } \\
\text { REGISTRO DE... }\end{array}$ \\
\hline \multicolumn{2}{|l|}{ Arranjo doméstico e potencial rede de suporte } \\
\hline $\begin{array}{l}1 \text { - Mora com outras pessoas na mesma casa? } \\
\text { Com quem? }\end{array}$ & $\begin{array}{l}\text { nome, tipo de relacionamento, sexo e idade das } \\
\text { pessoas que moram com o idoso }\end{array}$ \\
\hline 2 - Tem (outros) filhos? & nome, sexo, idade e onde residem os filhos \\
\hline 3 - Com que frequência se relaciona com: & frequências de relacionamentos com distintas pessoas \\
\hline $\begin{array}{l}4 \text { - Está satisfeito com a frequência com se relaciona } \\
\text { com: }\end{array}$ & $\begin{array}{l}\text { graus de satisfação do idoso quanto à frequência } \\
\text { com que se relaciona com distintas pessoas }\end{array}$ \\
\hline 5 - Como se sente com a relação que mantém com: & $\begin{array}{l}\text { graus de satisfação com relacionamentos com } \\
\text { distintas pessoas }\end{array}$ \\
\hline $\begin{array}{l}\text { 6- Tem alguém que cuida do sr quando fica doente? } \\
\text { Quem? }\end{array}$ & $\begin{array}{l}\text { nome, tipo de relacionamento, sexo e idade daqueles } \\
\text { que cuidam do idoso }\end{array}$ \\
\hline \multicolumn{2}{|l|}{ Perfil social } \\
\hline 7- Frequentou escola? Até que ano estudou? & grau de escolaridade do idoso \\
\hline 8 - Qual sua atividade profissional principal? & - \\
\hline 9 - Exerce essa atividade profissional atualmente? & - \\
\hline 10 - Tem trabalho remunerado atualmente? & - \\
\hline 11 - Quais são seus recursos financeiros atualmente? & recursos financeiros do idoso \\
\hline $\begin{array}{l}12 \text { - Com a sua situação econômica atual de que } \\
\text { forma satisfaz suas necessidades de alimentação, } \\
\text { moradia, saúde, etc? }\end{array}$ & $\begin{array}{l}\text { grau de satisfação de necessidades com situação } \\
\text { econômica atual }\end{array}$ \\
\hline \multicolumn{2}{|l|}{ Universo ocupacional } \\
\hline $\begin{array}{l}13 \text { - O que faz todos os dias, desde a hora acorda até } \\
\text { a hora que vai dormir? } \\
14 \text { - Tem alguma coisa que faça só de vez em quando? }\end{array}$ & atividades que compõe o cotidiano do idoso \\
\hline $\begin{array}{l}\text { 15 - Das coisas que faz, quais acha importante? } \\
\text { 15.1. Por quê? }\end{array}$ & atividades importantes e razões de importância \\
\hline $\begin{array}{l}\text { 16 - Das coisas que faz, quais gosta menos? } \\
\text { 16.1 Por quê? }\end{array}$ & atividades insatisfatórias e razões de insatisfação \\
\hline \multicolumn{2}{|l|}{ Capacidade Funcional } \\
\hline $\begin{array}{l}17 \text { - Tem dificuldade para fazer algumas das coisas } \\
\text { que faz? Que tipo(s) de dificuldade? } \\
18 \text { - O que faz quanto à dificuldade X: Encontrou um } \\
\text { jeito mais fácil de fazer, tem alguém que o(a) ajuda, faz } \\
\text { mesmo com dificuldade sem ajuda ou deixou de fazer? } \\
19 \text { - Caso tenha encontrado um jeito mais fácil de } \\
\text { fazer, como faz? Caso tenha alguém que o ajuda, } \\
\text { quem o ajuda? }\end{array}$ & $\begin{array}{l}\text { de atividades para quais o idoso tem dificuldade, sua } \\
\text { caracterização segundo tipos de dificuldade; níveis } \\
\text { de dificuldade e formas de compensação }\end{array}$ \\
\hline
\end{tabular}


Quadro 1 - Componentes do instrumento CICAc ${ }^{11}$. São Paulo, SP, 2008. (continuação)

\begin{tabular}{|c|c|}
\hline DIMENSÕES E QUESTÕES & $\begin{array}{l}\text { ACOMPANHA QUADRO PARA } \\
\text { REGISTRO DE... }\end{array}$ \\
\hline $\begin{array}{l}20 \text { - Pense nas coisas que faz com ajuda, com } \\
\text { dificuldade sem ajuda ou tenha deixado de fazer. } \\
\text { Imagina algum jeito mais fácil de fazê-las? } \\
\text { Sim, para todas as atividades ( ) } \\
\text { Sim, para algumas atividades () Não () } \\
21 \text { - Como poderia ser feito? }\end{array}$ & $\begin{array}{l}\text { Atividades para as quais apresenta dificuldade e } \\
\text { formas imaginadas de compensação }\end{array}$ \\
\hline $\begin{array}{l}22 \text { - Pense nas coisas que gostaria de ter feito em sua } \\
\text { vida mas nunca fez ou fez há muito tempo atrás e } \\
\text { gostaria de voltar a fazer. 22.1: Por que não faz? 22.2: } \\
\text { Imagina algum jeito de vir a fazê-las? }\end{array}$ & $\begin{array}{l}\text { de atividades desejadas, fatores de inibição e formas } \\
\text { imaginadas de realização }\end{array}$ \\
\hline
\end{tabular}

Segundo a OMS, ${ }^{1}$ foram definidos como critérios demográficos para enfermidades, incapacidades e morte: ter 80 anos e mais e ser mulher idosa solteira ou viúva; como critérios socioeconômicos: morar sozinho, estar socialmente isolado, não ter filhos e contar com recursos econômicos escassos Foram indicados em situação de risco funcional os idosos que requeriam ajuda especificamente para atividades básicas de vida diária. ${ }^{1}$

A capacidade funcional para atividades básicas e instrumentais foi aferida a partir da aplicação do instrumento CICAc. ${ }^{11,12}$ Quanto às atividades básicas, examinaram-se comportamentos em relação a alimentar-se, vestir-se, banhar-se, locomover-se e controlar eliminações. Para aferir a capacidade para atividades instrumentais, foram examinados comportamentos referentes a usar telefone, fazer compras, preparar refeições, realizar tarefas domésticas, tomar medicamentos e administrar finanças. ${ }^{11,12}$

Para cada atividade, básica e instrumental, os 190 idosos foram organizados em dois grupos, em relação à dificuldade para realizálas: os que não apresentavam dificuldade e os que apresentavam dificuldade para pelo menos uma atividade. Este último grupo foi organizado em três subgrupos correspondentes aos níveis de dificuldades: os que têm todas as suas dificuldades compensadas com práticas de autocuidado; ${ }^{8,9}$ aqueles que têm dificuldades não compensadas em uma ou mais atividades, mas não requerem ajuda; e por fim, os que requerem ajuda em uma ou mais atividade. ${ }^{11,12}$

Realizou-se descrição dos resultados em relação a presença ou não de critérios de riscos, ${ }^{1}$ presença ou não de dificuldades e para aqueles com dificuldades, sua distribuição segundo níveis. Foi conduzida comparação entre idosos que atendiam aos critérios demográficos e socioeconômicos para adoecimento, incapacidades e morte ${ }^{1}$ aqueles que não atendiam, em relação à proporção da necessidade de ajuda para atividades básicas e instrumentais de vida diária. Para tanto, utilizou-se o teste do $\chi^{2}$ adotando-se o nível significância de 5\%.

\section{RESULTADOS}

A análise das informações obtidas por meio da aplicação do CICAc ${ }^{11,12}$ permitiu caracterizar idosos quanto aos critérios de risco demográficos, socioeconômicos e funcionais, definidos pela OMS para adoecimento, incapacidades e morte, ${ }^{1}$ e comparar idosos que atendam ou não aos dois primeiros critérios em relação à necessidade de ajuda para atividades básicas e instrumentais de vida diária. 
Caracterização de idosos quanto aos critérios de risco demográficos, socioeconômicos e funcionais

Parcela importante dos participantes do estudo atende aos critérios de risco definidos pela OMS para adoecimento, incapacidades e morte. ${ }^{1}$ Quando a atenção a um ou mais critérios é considerada indistintamente, 152 $(80 \%)$ idosos estão submetidos; se dois ou três critérios são considerados, temos 86 (45\%) idosos nessa condição, conforme descrito na tabela 1.

Tabela 1 - Distribuição dos idosos segundo critérios de riscos¹. São Paulo, SP, 2008

\begin{tabular}{lcc}
\hline Idosos submetidos a critérios de risco & No & $\%$ \\
\hline Exclusivamente relativos à capacidade funcional & 4 & 2,6 \\
Exclusivamente sócio-econômicos & 15 & 9,9 \\
Exclusivamente demográficos & 47 & 30,9 \\
Dois ou três critérios & 86 & 56,6 \\
\hline Total de idosos submetidos a um ou mais critérios de riscos & 152 & 100,0 \\
\hline
\end{tabular}

Quando os critérios são considerados individualmente, identifica-se que $27,9 \%$ dos participantes têm 80 anos ou mais; $82 \%$ são mulheres, $70 \%$ não contam com a presença do cônjuge, seja pela perda do mesmo ou pela não constituição do vínculo conjugal ao longo da vida; $26,8 \%$ moram sozinhos, $23,7 \%$ não têm filhos; e 11,6\% não contam com ninguém que ajude em caso de necessidade. Apenas 4,7\% dos idosos consideram que, com sua situação econômica atual, satisfazem mal ou muito mal suas necessidades básicas.

As dificuldades para atividades básicas, que impliquem ou não ajuda, foram apontadas por 99 idosos (52,1\%). Deste, $57(57,6 \%)$ referem uma única dificuldade; $21(21,2 \%)$ referem duas; e a mesma proporção de idosos (21,2\%) aponta três ou mais dificuldades para essas atividades. Dentre os 99 idosos com dificuldades, 23 $(12.1 \%)$ compensam todas as suas dificuldades com práticas de autocuidado; $47(24,7 \%)$ não compensam uma ou mais dificuldades, porém não requerem ajuda; e $29(29,3 \%)$ requerem ajuda para pelo menos uma ABVD. Quando a totalidade de idosos participantes da pesquisa é considerada (190), a proporção de idosos que necessita de ajuda para as ABVDs é de 15,2\%.
Quanto às AIVDs, identificaram-se 92 idosos $(48,4 \%)$ com dificuldade para uma ou mais atividades. Destes, 49 (53,3\%) têm dificuldade para uma atividade; 25 (27,2\%), para duas e $18(19,6 \%)$ para três ou mais. Dentre os 92 idosos com dificuldades para atividades instrumentais, somente 10 (5,3\%) compensam todas as suas dificuldades com práticas de autocuidado; 15 (7,9\%) não compensam uma ou mais dificuldades, mas não requerem ajuda e $67(72,8 \%)$ requerem ajuda para pelo menos uma atividade. Quando a totalidade de idosos participantes da pesquisa é considerada (190), a proporção de idosos que necessita de ajuda para as atividades instrumentais é de 35,2\%.

A quase totalidade de idosos que têm dificuldade para as ABVDs identifica exclusivamente fatores intrínsecos relacionados, com predomínio de aspectos físicos e sensoriais. Somente um participante menciona fatores extrínsecos, ou relativos ao ambiente, para justificar suas dificuldades, e um menciona a combinação de fatores intrínsecos e extrínsecos. À semelhança do que ocorre para as ABVDs, dentre os idosos com dificuldades para AIVDs, somente quatro $(4,3 \%)$ referem fatores extrínsecos ou combinados para explicarem suas 
dificuldades. E ao se considerar o conjunto das atividades de vida diária, básicas e instrumentais, 130 idosos $(68,4 \%)$ apresentaram dificuldades, sendo que $102(78,4 \%)$ apresentaram uma ou duas e 28 idosos $(21,5 \%)$, três ou mais.

Através da aplicação do instrumento CICAc, ${ }^{11,12}$ pode-se identificar que as práticas de autocuidado ${ }^{8,9}$ constituíram-se como possibilidade de enfrentamento de dificuldades por alguns idosos e não por outros. Entre os que adotam práticas para o conjunto de suas atividades, 16 idosos $(8,4 \%)$ o fazem para todas suas dificuldades; $38(20 \%)$ as adotam para compensar parte dessas dificuldades, realizando as demais atividades mesmo com dificuldade, porém sem ajuda; enquanto $76(40 \%)$ requerem ajuda. Com base na ausência ou presença de dificuldades e nas formas de enfrentamento dessas, são previstos níveis de independência, de acordo com tabela 2 .

Tabela 2 - Distribuição dos idosos segundo níveis de dificuldades para atividades básicas e instrumentais de vida diária e práticas de autocuidado. São Paulo, SP, 2008.

\begin{tabular}{lcc}
\hline Níveis de dificuldade & $\mathrm{N}$ & $\%$ \\
\hline Atividades básicas de vida diária & & \\
ND & 91 & 47,9 \\
DC & 23 & 12,1 \\
DNC & 47 & 24,8 \\
RA & 29 & 15,2 \\
\hline Total & 190 & 100,0 \\
\hline Atividades instrumentais de vida diária & & \\
ND & 98 & 51,6 \\
DC & 10 & 5,3 \\
DNC & 15 & 7,9 \\
RA & 67 & 35,2 \\
\hline Total & 190 & 100,0 \\
\hline
\end{tabular}

Legenda ND: Não tem Dificuldade em nenhuma atividade; DC: Tem todas as suas Dificuldades Compensadas com práticas de autocuidado; DNC: Tem Dificuldades Não Compensadas em uma ou mais atividades, mas não requer ajuda; RA: Requer Ajuda em uma ou mais atividade.

Pode-se afirmar, com base na tabela 2, que um pequeno percentual de idosos adota práticas de autocuidado tanto no que se refere às ABVDs (12,1\%), quanto às AIVDs (5,3\%). Acrescenta-se que 6,3\% adotam práticas para compensar somente parte de suas dificuldades nas ABVDs e 4,8\%, para compensar parcela de suas dificuldades nas AIVDs. As atividades básicas para as quais um maior percentual de idosos refere usar essa estratégia foram controlar eliminações (35\%) e locomover-se $(27 \%)$. As atividades instrumentais para as quais um maior percentual de idosos adota práticas efetivas de autocuidado foram fazer compras $(23 \%)$ e realizar tarefas domésticas (11\%). Os idosos, quando adotam práticas, geralmente lançam mão de modificações comportamentais, seguidas por adoção de dispositivos auxiliares e raramente dirigem-se ao ambiente, conforme está exemplificado no quadro 2. 
Quadro 2 - Exemplos de práticas de autocuidado para atividades de vida diária, São Paulo, SP, 2008

\begin{tabular}{|c|c|c|c|}
\hline Atividades & Modificações Comportamentais & $\begin{array}{l}\text { Modificações } \\
\text { Ambientais }\end{array}$ & Dispositivos auxiliares \\
\hline Banho & $\begin{array}{l}\text { "esfregar um pé no outro" por } \\
\text { dificuldade em alcançar e lavar os } \\
\text { pés, "segurar-se na janela" e "tomar } \\
\text { banho mais cedo para aproveitar a } \\
\text { iluminação natural" }\end{array}$ & & $\begin{array}{l}\text { "sentar-se em banquinho } \\
\text { antiderrapante no box", } \\
\text { "usar cadeira de banho" e } \\
\text { "adotar barras de segurança } \\
\text { no banheiro". }\end{array}$ \\
\hline Vestuário & $\begin{array}{l}\text { "utilizar o membro não afetado", } \\
\text { "apoiar-se na mureta" e "sentar-se na } \\
\text { cama" }\end{array}$ & $\begin{array}{l}\text { "manter roupas } \\
\text { sempre no } \\
\text { mesmo lugar" }\end{array}$ & \\
\hline Continência & "ingerir menos água a noite" & & $\begin{array}{l}\text { "adoção de protetor de } \\
\text { calcinha, absorvente } \\
\text { ou fralda geriátrica", } \\
\text { "tratamento médico e } \\
\text { fisioterápico" }\end{array}$ \\
\hline Alimentação & & & "adaptação para colher" \\
\hline Locomoção & $\begin{array}{l}\text { "descer escadas sentada", "proteger-se } \\
\text { de obstáculos com mão à frente do } \\
\text { rosto (pessoa com deficiência visual)", } \\
\text { "parar para descansar", "evitar sapato } \\
\text { com salto". }\end{array}$ & $\begin{array}{l}\text { "arranjar o } \\
\text { ambiente } \\
\text { de modo a } \\
\text { apoiar-se em } \\
\text { móveis estáveis } \\
\text { enquanto anda" }\end{array}$ & $\begin{array}{l}\text { "uso de bengala", } \\
\text { "joelheira", "andador" e } \\
\text { "prótese" }\end{array}$ \\
\hline Uso de telefone & "gravar números de memória" & & $\begin{array}{l}\text { "utilizar aparelho auditivo" } \\
\text { ou "lupa". }\end{array}$ \\
\hline Compras & $\begin{array}{l}\text { "fracionar as compras","usar carrinho } \\
\text { de feira". }\end{array}$ & & \\
\hline $\begin{array}{l}\text { Tarefas } \\
\text { domésticas }\end{array}$ & $\begin{array}{l}\text { "parar para descansar", "fazer aos } \\
\text { poucos", "fazer lentamente", "realizar } \\
\text { algumas atividades na posição } \\
\text { sentada" }\end{array}$ & & $\begin{array}{l}\text { "tomar medicação } \\
\text { recomendada para dor nas } \\
\text { costas". }\end{array}$ \\
\hline $\begin{array}{l}\text { Tomar } \\
\text { medicamentos }\end{array}$ & $\begin{array}{l}\text { "lembretes na porta da geladeira", } \\
\text { "marcação de tomadas de } \\
\text { medicamento em um caderninho", } \\
\text { "sistematização de horário para tomar } \\
\text { os medicamentos", "medicamentos são } \\
\text { agrupados em local de fácil acesso" }\end{array}$ & & \\
\hline
\end{tabular}


A análise estatística permitiu comparar grupos de idosos que atendiam, ou não, aos critérios demográficos e socioeconômicos de risco previstos pela $\mathrm{OMS}^{1}$ segundo a necessidade, ou não, de ajuda para atividades básicas e instrumentais.

Tabela 3 - Distribuição de idosos segundo critérios demográficos e socioeconômicos de risco definidos pela $\mathrm{OMS}^{1}$ e necessidade de ajuda para atividades básicas e instrumentais de vida diária. São Paulo, SP, 2008

\begin{tabular}{lcccc}
\hline Critérios de Risco & $\begin{array}{c}\text { Não atende } \\
\text { a nenhum } \\
\text { critério }\end{array}$ & $\begin{array}{c}\text { Atende a } \\
\text { um ou dois } \\
\text { critérios }\end{array}$ & Total & $\chi^{2}$ p
\end{tabular}

Níveis de dificuldade

\begin{tabular}{lcccc}
\hline Atividades básicas de vida diária & & & & \\
NRA & 38 & 123 & 161 & \\
RA & 0 & $25^{*}$ & 25 & \\
Total & 38 & 148 & 186 & $\begin{array}{c}\chi^{2} 7,52 \\
\mathrm{p}<0,01\end{array}$ \\
\hline Atividades instrumentais de vida diária & & & & \\
NRA & 34 & 89 & 123 & \\
RA & 4 & 63 & 67 & \\
\hline Total & 38 & 152 & 190 & $\chi^{2}=12,73$ \\
& & & & $\mathrm{p}<0,01$ \\
\hline
\end{tabular}

Nota* do total de 29 idosos que requerem ajuda para ABVDs foram excluídos 4 casos que respondiam especificamente a esse critério de risco Legenda: NRA: Não requer ajuda; RA: Requer ajuda

Como se observa na tabela 3, o grupo de idosos que atende a um ou dois critérios de risco ${ }^{1}$ apresenta proporção maior de indivíduos que requerem ajuda para atividades básicas e instrumentais de vida diária.

\section{DISCUSSÃO}

Uma proporção significativamente maior de mulheres (82\%) do que homens participaram da pesquisa, o que corrobora dados obtidos em outros estudos. ${ }^{13,14,15}$ A feminização do envelhecimento é um dos grandes desafios a serem enfrentados como decorrência do envelhecimento populacional. Em quase todos os lugares do mundo, as mulheres vivem mais do que os homens, correspondendo aproximadamente a dois terços da população acima de 75 anos em países como Brasil e África do Sul. ${ }^{2}$

A maioria dos idosos pesquisados não conta com a presença do cônjuge ( $70 \%$ ) e compreendem predominantemente viúvos $(48,9 \%)$, solteiros $(17,4 \%)$ e divorciados (4,2\%). Esse dado se justifica, mais uma vez, pela maior concentração de mulheres do que homens no estudo e pelo fato de estas se manterem viúvas em maior proporção do que aqueles. Reconhece-se que a proporção de mulheres viúvas é maior do que a de homens, porque, além da tendência dos homens de casarem com mulheres mais jovens e de contraírem novo matrimônio após o falecimento de suas esposas, a expectativa de vida das mulheres é maior quando comparada à dos homens. ${ }^{2}$ 
Observa-se que uma proporção expressiva de idosos pesquisados mora sozinha $(26,8 \%)$, superando aquela obtida em outros estudos, que encontraram $21,2 \%$ e $14.5 \%$ de idosos nessa condição. 14,15 "Morar só" pode representar um risco para o idoso, dependendo sobretudo de suas condições funcionais e de características de sua rede de suporte social. Associação entre morar sozinho e alta mortalidade foi estabelecida em estudo conduzido por Lee. ${ }^{7}$

Um percentual de $23,7 \%$ idosos não tem filhos, o que pode implicar risco a vida, saúde e funcionalidade dos idosos, pois a família é o vínculo primeiro a ser acionado quando da instalação de alguma incapacidade e o principal suporte para a não-institucionalização do idoso. Com o passar dos anos, a relação entre idosos e filhos adquire nova dinâmica, quando aumenta a função dos filhos como cuidadores dos pais. ${ }^{16-}$

Não contar com ninguém que ajude sugere isolamento social, o que, por sua vez, implica grande vulnerabilidade do idoso, ameaçando sua permanência no domicílio e até mesmo sua vida. Resultados de pesquisa desenvolvida ${ }^{17}$ indicam um aumento de 50\% na probabilidade de sobrevivência de idosos com fortes relações sociais, após controle de variáveis como idade, sexo e estado de saúde.E embora esta condição tenha sido referida somente por $11,6 \%$ dos entrevistados, ela se reveste de significado, suscitando especialmente com outras informações como "morar só" e "não ter filhos", reflexão sobre estratégias para mapeamento, potencialização e ampliação da rede de suporte social desses idosos.

A porcentagem expressiva de idosos pesquisados, que atende a um ou mais critérios de riscos sociais previstos pela OMS, ${ }^{1}$ corrobora dados da literatura. Aponta-se o idoso como vulnerável ao enfraquecimento e diminuição de sua rede. Essa sofre mais perdas, os vínculos existentes diminuem por morte e pelas reduzidas oportunidades de substituição dos mesmos. Os idosos se veem com menos amigos e cada vez mais recolhidos e restritos às relações familiares, o que indica a importância de políticas públicas que apoiem a atenção familiar prestada aos idosos. ${ }^{16,18}$ A perda ou não constituição da relação conjugal, frequente entre os pesquisados, pode significar menor efetividade ainda de sua rede de suporte social.

A prevalência de dificuldades para atividades básicas (52,3\%); instrumentais $(48,2 \%)$ e, consequentemente, para o conjunto dessas atividades (68,4\%), supera a encontrada em estudos sobre capacidade funcional no Brasil, na década de 90 , que variou de $28 \%$ a $61 \% .{ }^{19} \mathrm{~A}$ maior prevalência de incapacidades no presente estudo está possivelmente relacionada à grande proporção de participantes acima de 75 anos, uma vez que as incapacidades aumentam conforme a idade avança. ${ }^{714,20}$

Considerando-se a totalidade dos participantes da pesquisa, 15,2\% requerem ajuda para pelo menos uma atividade básica de vida diária (ABVD) e 67 idosos $(35,2 \%)$ requerem ajuda para pelo menos uma AIVD. Esses dados aproximam-se daqueles obtidos em estudo conduzido no Brasil por Lebrão e Duarte, ${ }^{21}$ que encontrou $19,2 \%$ de idosos que necessitam de ajuda nas ABVDs e, 26,8\% nas AIVDs; são inferiores ao estudo desenvolvido por Nakatani et al., ${ }^{22}$ que encontraram prevalência de incapacidades de 40,9\% para as básicas e $58 \%$, para as instrumentais e, superiores a dados obtidos por Pacheco ${ }^{13}$, uma vez que este identificou aproximadamente $15 \%$ idosos que requerem ajuda para o conjunto das atividades básicas e instrumentais de vida diária.

Buscou-se identificar, através desta pesquisa, além da presença, fatores relacionados às dificuldades, níveis de dificuldade e práticas de autocuidado para seu enfrentamento. A quase totalidade de idosos identifica fatores exclusivamente intrínsecos para suas dificuldades nas ABVDs. Admite-se a supremacia dos fatores intrínsecos sobre os extrínsecos quando se trata destas atividades, já que a competência para elas está fortemente relacionada aos recursos relativos à saúde. ${ }^{20}$ Limitações que potencialmente fragilizam a capacidade funcional dos idosos podem incluir tontura, instabilidade, 
diminuição da acuidade auditiva e visual, entre outros. ${ }^{13}$ Acrescenta-se que foram estabelecidas associações estatisticamente significantes entre idade, acuidade visual diminuída, doenças associadas, depressão, equilíbrio e mobilidade à dependência dos idosos. ${ }^{22}$

À semelhança das ABVDs, os participantes identificam quase que exclusivamente a importância dos fatores intrínsecos para suas dificuldades nas AIVDs, desconsiderando a complexidade que cerca a realização dessas atividades. A capacidade funcional é produto da interação de fatores intrínsecos e extrínsecos ${ }^{23}$ e especialmente no que se refere às atividades instrumentais e outras atividades produtivas, a competência está fortemente relacionada aos recursos sociais, psicológicos e comportamentais. $^{20}$

Dentre as práticas de autocuidado ${ }^{8,9}$ adotadas para enfrentamento de dificuldades, identificaram-se modificações comportamentais, seguida por adoção de dispositivos auxiliares e modificações ambientais. $\mathrm{O}$ uso de técnicas adaptadas tem sido apontado como preferível ao uso de equipamentos, pois as técnicas conferem à pessoa maior flexibilidade enquanto equipamentos implicam em custo, reparos, transporte e aceitação por parte do usuário.

Embora as modificações ambientais também se constituam em estratégias compensatórias e algumas sejam pouco onerosas, foram adotadas menos frequentemente pelos idosos. Esse dado se apoia em parte na dificuldade dos idosos em identificar fatores ambientais como geradores de incapacidades e corrobora a afirmação de Cummings et al., ${ }^{24}$ de que pessoas mais velhas não acreditam que modificações ambientais possam diminuir incidência de quedas e fraturas.

A idade avançada esteve associada à capacidade funcional em outros estudos, ${ }^{14,21,25}$ assim como "ser mulher" 21 e "avaliar sua situação econômica como razoável, ruim ou muito ruim". ${ }^{25}$ Estes resultados reafirmam associações, encontradas ${ }^{26}$ entre capacidade funcional e fatores demográficos, socioeconômicos, culturais e psicossociais.

\section{CONCLUSÃO}

Através da pesquisa realizada nos domicílios, constatou-se que um número expressivo de idosos pesquisados atende a maior parte dos critérios de risco definidos no estudo, ou seja, demográficos, socioeconômicos e funcionais. ${ }^{1}$ $\mathrm{Na}$ comparação entre grupos, identificou-se uma proporção maior de idosos que requerem ajuda nas atividades cotidianas e atendem aos critérios demográficos e/ou socioeconômicos de risco ${ }^{1}$ ( $\mathrm{p}$ $<0,05)$ em relação ao grupo que requer ajuda e não atende a esses critérios.

Embora o estudo permita identificar um número significativamente maior de idosos que requer ajuda para atividades básicas e instrumentais e atende a um ou dois critérios de riscos ${ }^{1}$, outras análises deverão ser conduzidas para verificar a influência específica de cada um desses critérios sobre a funcionalidade.

O pequeno número de idosos que adota práticas de autocuidado inviabilizou comparação entre grupos, que empregam ou não essas práticas, e critérios de risco. ${ }^{1}$ Realização de pesquisa sobre essas práticas com um maior número de indivíduos poderá ser conduzida, a fim de possibilitar essa análise.

Apesar das limitações do estudo, ele suscita planejamento de ações de promoção da saúde abrangentes. Essas ações devem voltar-se tanto aos idosos que têm dificuldade, mas ainda não requerem ajuda, quanto a aqueles que requerem auxílio para suas atividades cotidianas. Os idosos na primeira condição têm potencial para desenvolver fragilidade e por isso, de acordo com a Política Nacional de Saúde da Pessoa Idosa, ${ }^{3}$ merecem atenção específica e frequente dos profissionais de saúde. Os idosos que requerem ajuda são considerados frágeis e suscitam ações de reabilitação. $\mathrm{O}$ grupo de idosos que atende os critérios de risco social e necessita de ajuda encontra-se em situação de vulnerabilidade, suscitando ações complexas voltadas ao mapeamento, potencialização e ampliação de redes sociais, prevenção, controle ou reversão de dependências. 


\section{REFERÊNCIAS}

1. Morales Martínez F. Evaluación integral del anciano. In: Anzola Perez E, Galinsk D, Morales e Martinez F, Solas AR, Sánchez Ayéndez M. La atención de los ancianos: un desafio para los años noventa. Washington (DC): Organizacion Panamericana de la Salud; 1994.

2. World Health Organization. Envelhecimento ativo: uma política de saúde. Brasília: Organização PanAmericana de Saúde; 2005.

3. BRASIL. Portaria n. 2.528, de 19 de outubro de 2006. Aprova a Política Nacional de Saúde da Pessoa Idosa. Brasília: 2006. Disponível em: http:// bvsms.saude.gov.br/bvs/saudelegis/gm/2008/ ptr2528_22_10_2008.html

4. Litvoc J. El envejecimiento de la población: un desafio que va más allá del año 2000. Bol Oficina Sanit Panam 1990; 109 (1): 1-5.

5. BRASIL. Portaria n. 1.395, de 9 de dezembro de 1999. Aprova a Política Nacional de Saúde do Idoso e dá outras providências. Diário Oficial da República Federativa do Brasil, Brasília (DF). 199913 dez; Seção I: 237.

6. Restrepo HE, Anzola Perez E. Promoción de la salud de los ancianos. In: Anzola Perez E, Galinsk

D, Morales e Martinez F, Solas AR, Sánchez Ayéndez M. La atención de los ancianos: un desafio para los años noventa. Washington (DC): Organizacion Panamericana de la Salud; 1994. p. 383-8.

7. Lee Y. The predictive value of self assessed general, physical, and mental health on functional decline and mortality in older adults. J Epidemiol Community Health 2000; 54 (2):123-9.

8. Norburn JE, Bernard SL, Konrad TR, Woomert A, DeFriese GH, Kalsbeek WD, et al. Self-care and assistance from others in coping with functional status limitations among a national sample of older adults. J Gerontol B Psychol Sci Soc Sci 1995; 50 (2): S101-9.

9. Gitlin LN, Winter L, Dennis MP, Corcoran M, Schinfeld S, Hauck WW. Strategies used by families to simplify tasks for individuals with alzheimer's disease and related disorders: Psycometric Analysis of Task Management Strategy Index (TMSI). Gerontologist 2002; 42 (1): 61-9.

10. Mann WC, Hurren D, Machiko T. Assistive devices used by home-based elderly persons with arthrits. Am J Occup Ther 1995; 49 (8): 810-20.

11. Almeida MHM, Spinola AWP, Lancman S. Técnica Delphi: validação de um instrumento para uso do terapeuta ocupacional em gerontologia. Rev. Ter. Ocup. Univ. São Paulo 2009; 20 (1): 49-58.
12. Almeida MHM, Spínola AWP, Iwamizu OS, Okura RIS, Barriso LP, Lima ACP. Confiabilidade do Instrumento para Classificação de Idosos quanto à Capacidade para o Autocuidado. Rev Saúde Pública 2008; 42(2): 317-23.

13. Pacheco RO, Santos SSC. Avaliação global de idosos em unidades de PSF. Textos sobre Envelhecimento 2004; 7(2).

14. Tavares DMS, Pereira GA, Iwamoto HH, Miranzzi SSC, Rodrigues LR, Machado ARM. Incapacidade funcional entre idosos residentes em um município do interior de Minas Gerais. Texto Contexto Enferm 2007; 16(1): 32-9.

15. Souza L, Galante H, Figueredo D. Qualidade de vida e bem-estar dos idosos: um estudo exploratório na população portuguesa. Rev Saúde Pública 2003; 37 (3): 364-71.

16. Sluzki CE. A rede social na prática sistêmica: alternativas terapêuticas. São Paulo: Casa do Psicólogo; 1997.

17. Holt-Lunstad J, Smith TB, Layton JB. Social relationships and mortality risk: a meta-analytic review. PLoS Medicine 2010; 7(7): e1000316.

18. Organização Pan-Americana da Saúde. Relatório Final. OPAS- 25a Conferência Sanitária PanAmericana e 50a Sessão do Comitê Regional. Washington (DC); 1998 Set 21-25.

19. Litvoc J, Derntl AM. Capacidade funcional do idoso: significado e aplicações. In: Cianciarullo TI, Gualda DMR, Silva GTR, Cunha ICKO. Saúde na família e na comunidade. São Paulo: Robe editorial; 2002. p.268-319.

20. Baltes MM, Mayr U, Borchelt M, Maas I, Wilms HU. Everyday competence in Old and very old age: an inter-disciplinary perspective. Ageing and Society 1993; 13 (4): 657-80.

21. Duarte YAO. Desempenho funcional e demandas assistenciais. In: Lebrão ML, Duarte YAO. Saúde, bemestar e envelhecimento/SABE. Brasília: Organização Pan-Americana da Saúde; 2003. p. 83-200.

22. Nakatani AYK, Costa EFA, Teles SA, Silva LB, Rêgo MAB, Silva-e-Souza AC, et al. Perfil sóciodemográfico e avaliação funcional de idosos atendidos por uma equipe de saúde da família na periferia de Goiânia, Goiás. Rev Soc Bras Clín Méd 2003; 1(5): 131-6.

23. Kane RL. The implication of assessment. J Gerontol 1993; 48 (Sp.Issue): 27-31. 
24. Cumming RG, Thomas M, Szonyi G, Frampton G, Salkeld G, Clemson L. Adherence to occupational therapist recommendations for home modifications for falls prevention. Am J Occup Ther 2001; 55 (6): 641-8.

25. Fiedler MM, Peres KG. Capacidade funcional e fatores associados em idosos do Sul do Brasil: um estudo de base populacional. Cad. Saúde Pública 2008; 24(2): 409-15.

26. Rosa TEC, Benício MHD, Latorre MRDO, Ramos LR. Fatores determinantes da capacidade funcional entre idosos. Rev Saúde Pública 2003; 37(1): 40-8.

Recebido: 21/2/2011

Reapresentado: 10/9/2011

Aprovado: 13/12/2011 